\title{
La lengua rumana en Madrid: observaciones sobre usos y actitudes lingüísticas
}

\author{
Diego MuÑOZ CARROBLES \\ Departamento de Filología Románica, Fil. Eslava y Ling. Gral. \\ Universidad Complutense de Madrid \\ dmcarrobles@fillol.ucm.es
}

\begin{abstract}
RESUMEN
En este trabajo trataremos un caso reciente de lenguas en contacto en el ámbito románico: la lengua rumana que se habla en Madrid y en su área metropolitana como consecuencia del intenso flujo migratorio procedente de Rumanía que esta zona ha experimentado desde los primeros años del siglo XXI. Pretendemos dar a conocer el estado actual del proceso de contacto entre las dos lenguas a través del testimonio de los propios hablantes. Por un lado, analizaremos los dominios en que los inmigrantes rumanos siguen usando su lengua: tiempo libre, familia, trabajo, etc. Por otra parte, queremos dar a conocer las actitudes de los encuestados hacia su lengua primera, con el fin de comprobar si asistimos a un proceso de mantenimiento o de sustitución de la lengua.
\end{abstract}

Palabras clave: lenguas en contacto, lengua rumana, actitudes, mantenimiento, sustitución

[Recibido, julio 2010; aprobado, noviembre 2010]

The Romanian language in Madrid:

some remarks on the linguistic uses and attitudes

\begin{abstract}
In this paper we will take care of a recent case of language contact: the Romanian language spoken in Madrid and its metropolitan area, as a consequence of the intense migration from Romania to this particular region of Spain, during the first years of the $21^{\text {st }}$ century. We aim to show the current state of the contact process through the speakers' experiences. On one hand, we will analyze the contexts in which Romanian immigrants keep using their language: free time, family, work. On the other hand, we want to check the speakers' attitudes, in order to know if we are facing language shift or language maintenance.
\end{abstract}

Keywords: languages in contact, Romanian language, attitudes, maintenance, shift.

\section{Presentación}

La intención de este trabajo es acercarnos a la realidad sociolingüística de la inmigración rumana instalada en la Comunidad de Madrid, un colectivo que agrupa ya a más de doscientas mil personas y que ha experimentado un crecimiento exponencial en los últimos cinco años. Este fenómeno social ha provocado, entre otras 
consecuencias, que se produzca el contacto entre la lengua de la sociedad de acogida, el español, y la lengua de los recién llegados, el rumano.

En primer lugar, describiremos brevemente la comunidad rumana de Madrid, proporcionando datos recientes y posteriormente expondremos el cuestionario empleado para realizar esta investigación y que hemos distribuido entre diferentes miembros de la citada comunidad de habla. Aquél abarca distintos puntos relacionados con la utilización de la lengua rumana por parte de sus hablantes así como las actitudes de la población rumana con respecto a su lengua y al español.

Así mismo centraremos nuestra atención en las actitudes y los usos lingüísticos de los rumanos establecidos en Madrid. Se trata de proporcionar una serie de claves para interpretar cuándo, cómo y por qué los rumanos deciden emplear su lengua o sustituirla. En definitiva, esperamos proporcionar una imagen global de la realidad sociolingüística de la comunidad rumana de Madrid a partir del testimonio directo de sus miembros.

\section{Datos generales: la presencia rumana en la Comunidad de Madrid}

Sin duda el espectacular crecimiento de la inmigración rumana en España y concretamente en la Comunidad de Madrid es uno de los fenómenos sociales más destacables de los últimos años en España. La presencia de rumanos en el área de Madrid empezó a crecer desde finales de la década de los 90, en torno a tres centros principales: Alcalá, Coslada y Arganda, que siguen siendo hoy día tres núcleos significativos de presencia rumana en Madrid.

Si utilizamos los datos del Padrón a 1 de enero de 2009, elaborados por el Instituto Nacional de Estadística ${ }^{1}$ y referidos al conjunto del Estado español, veremos cómo la comunidad rumana roza los ochocientos mil miembros -casi un $2 \%$ de la población española es ya de origen rumano. Los ciudadanos de nacionalidad rumana constituyen pues, en 2009, la comunidad extranjera más numerosa en España.

\begin{tabular}{|l|l|l|}
\hline España & 46.157 .822 & $100 \%$ \\
\hline Total Extranjeros & 5.598 .691 & $12^{\prime} 13 \%$ \\
\hline Rumanos & 796.576 & $1^{\prime} 72 \%$ \\
\hline
\end{tabular}

Tabla 1: Población total extranjera y rumana en España, 2009.

Elaboración propia, a partir de datos del Instituto Nacional de Estadística. ${ }^{2}$

De nuevo el grupo más numeroso dentro de los extranjeros residentes en la Comunidad de Madrid lo constituyen los ciudadanos rumanos, que suman más de dos-

${ }^{1}$ Los datos pertenecientes al Instituto Nacional de Estadística que forman parte de esta investigación han sido obtenidos a través de la página web: $w w w$.ine.es

${ }^{2}$ Datos referidos al avance de resultados del Padrón a 1 de enero de 2009. 
cientas mil personas, cifra que supone una proporción cercana a la quinta parte del total de la población extranjera.

\begin{tabular}{|l|l|l|}
\hline Comunidad de Madrid & 6.360 .241 & $100 \%$ \\
\hline Total Extranjeros & 1.043 .113 & $16^{\prime} 41 \%$ \\
\hline Rumanos & 200.610 & $3^{\prime} 15 \%$ \\
\hline
\end{tabular}

Tabla 2: Población total y extranjera en la Comunidad de Madrid, 2009

Elaboración propia, a partir de datos del Instituto Nacional de Estadística

Ahora bien, la distribución de estos habitantes no es necesariamente uniforme. Hay muchos más rumanos en Madrid capital y en la zona llamada Corredor del Henares -parte de la corona metropolitana madrileña que se extiende alrededor de la autovía A2 en dirección NE y que incluye municipios como Coslada, San Fernando de Henares, Torrejón de Ardoz y Alcalá de Henares. También hay una importante colonia rumana en la zona sureste, en torno a Arganda del Rey y RivasVaciamadrid, así como en los grandes municipios del cinturón sur de la Comunidad (Getafe, Móstoles, Alcorcón, Fuenlabrada, Leganés, Parla...).

\section{El 'Cuestionario sobre actitudes lingüísticas'}

La parte empírica de este trabajo de investigación la hemos llevado a cabo mediante la elaboración del "Cuestionario sobre las actitudes lingüísticas de la comunidad rumana de Madrid". Se trataba de ver, a partir de una serie de preguntas breves, cuáles son las principales actitudes de los rumanos encuestados sobre su propia lengua, cómo, cuándo y con quién la usan, así como cuáles son sus opiniones sobre el futuro del idioma. El cuestionario fue elaborado en español, para evitar la posible solidaridad de los informantes con un documento elaborado en su lengua primera, lo cual podría haber provocado la aparición de respuestas menos ajustadas a la realidad.

En total dispusimos de 87 cuestionarios completos, procurando atender a la realidad demográfica de la comunidad rumana de Madrid, es decir, prestando especial atención al grupo de edad correspondido entre los 18 y los 45 años, que representa la franja de edad mayoritaria, según los datos del Padrón. El acceso a los informantes se realizó de manera directa e indirecta, gracias a la colaboración de asociaciones rumanas como RoMadrid y del CEPI de Alcalá de Henares y de Coslada.

\begin{tabular}{|l|l|}
\hline Grupo de edad & Población \\
\hline $0-15$ años & 17.976 \\
\hline $16-44$ años & 139.752 \\
\hline $45-64$ años & 23.298 \\
\hline 65 años y más & 1.004 \\
\hline
\end{tabular}

Tabla 3: Población rumana por grandes grupos de edad.

Elaboración propia, a partir de datos obtenidos del Instituto Nacional de Estadística 
La distribución entre hombres y mujeres difiere de los datos del INE, ya que más mujeres que hombres respondieron a nuestro cuestionario, mientras que las cifras de población dan cuenta de una población masculina levemente superior a la femenina, tanto en la franja de población activa como en otras. Por edades si se respeta la proporción con los datos oficiales, ya que $3 / 4$ partes de los informantes pertenecen al grupo de edad comprendido entre 18 y 45 años.

\begin{tabular}{|l|l|}
\hline \multicolumn{2}{|c|}{ Sexo } \\
\hline Mujeres & $58^{\prime} 62 \%$ \\
\hline Hombres & $41^{\prime} 37 \%$ \\
\hline
\end{tabular}

Tabla 4: Distribución de los informantes por sexo.

Resultados del 'Cuestionario sobre actitudes lingüísticas...'

\begin{tabular}{|l|l|l|l|l|}
\hline \multicolumn{1}{|c|}{ Grupo de edad } & \multicolumn{2}{|c|}{ Mujeres } & \multicolumn{2}{c|}{ Hombres } \\
\hline $\begin{array}{l}\text { Edad escolar } \\
\text { (de 0 a 15 años) }\end{array}$ & 8.760 & $48^{\prime} 86 \%$ & 9.166 & $51^{\prime} 13 \%$ \\
\hline $\begin{array}{l}\text { Población activa } \\
\text { (de 16 a 64 años) }\end{array}$ & 77.805 & $47^{\prime} 71 \%$ & 85.245 & $52^{\prime} 28 \%$ \\
\hline
\end{tabular}

Tabla 5: Población rumana de la Comunidad de Madrid por sexo y grupos de edad. Elaboración propia, a partir de datos obtenidos del Instituto Nacional de Estadística

\begin{tabular}{|l|l|}
\hline \multicolumn{2}{|c|}{ Edad } \\
\hline de 18 a 25 & $21^{\prime} 81 \%$ \\
\hline de 25 a 30 & $25^{\prime} 45 \%$ \\
\hline de 30 a 40 & $29^{\prime} 09 \%$ \\
\hline de 40 a 50 & $7^{\prime} 27 \%$ \\
\hline más de 50 & $166^{\prime} 36 \%$ \\
\hline
\end{tabular}

Tabla 6 Distribución de los informantes por edad.

Resultados del 'Cuestionario sobre actitudes lingüísticas...'

La primera parte del cuestionario se ocupa de los datos personales del informante. En esta primera parte (A. Datos personales) la intención era separar y clasificar por variables extralingüísticas los datos que obtendremos en el análisis de los cuestionarios. Los dos primeros factores son esenciales en nuestro estudio: el sexo y la edad de los informantes; aunque hemos de recordar que estas variables sociales no son universales, sino específicas y que factores como el nivel de instrucción, el sexo/género, la ocupación actual y la edad pueden funcionar de manera diferente en comunidades de habla distintas. Nosotros pensamos que era necesario ajustar 
la edad y el sexo de los informantes a los datos generales de población de que disponíamos.

Pasamos a comentar la sección C de nuestro cuestionario, llamada "Usos lingüísticos", cuya finalidad es ver en cuál de las dos lenguas en contacto -rumano y español- se desarrollan los diferentes ámbitos de la comunicación o bien cuál de las dos variedades es la empleada para dirigirse a diferentes interlocutores, como familia o amigos y, por supuesto, la transmisión a los hijos. Para ello hemos diseñado una escala de cinco grados, que van desde la utilización exclusiva de una o otra lengua, hasta el grado intermedio de alternancia social de idiomas ${ }^{3}$. En nuestra escala de 1 a 5, la puntuación 1 correspondería al valor "Español"; la 2, a "más en español que en rumano"; la puntuación 3, a "tanto en rumano como en español"; la 4, a "más en rumano que en español"; y por último la puntuación 5 corresponde a "Rumano".

Ahora bien, en ocasiones las preguntas están deliberadamente desordenadas para evitar obtener la misma respuesta en todos los casos. Se pide, por un lado, que hagan una valoración sobre un hecho general (\#17) y por otro lado, una valoración sobre decisiones personales propias (\#18) o ajenas (\#19).

De hecho todo un apartado del cuestionario se refiere a la transmisión de la lengua, aspecto esencial de los usos lingüísticos de una comunidad de habla inmigrante. En algunas preguntas hemos incorporado la posibilidad de que el informante añadiera más información y respondiera a la pregunta ‘¿Por qué?'. El objetivo principal de esta sección es saber si los rumanos desean transmitir la lengua rumana a la siguiente generación y conocer su opinión sobre el futuro de la lengua, así como sobre las medidas de planificación necesarias para su conservación.

En cuanto a los ámbitos que los informantes debían valorar se refieren a las relaciones familiares, de amistad, de trabajo y algunas necesidades comunicativas sociales. También queríamos saber si el rumano sigue siendo utilizado en las actividades de ocio. Esto no solamente nos proporciona una información sobre el estilo de vida de la comunidad de habla, sino que también nos indica si los hablantes siguen expuestos al rumano escrito, a la norma culta. Nos parece importante, en el caso de colectivos inmigrantes, destacar la relación entre la lectura en la lengua primera y la conservación de la lengua. Si se corta esta relación se abre más fácilmente el camino hacia las interferencias, los préstamos en masa y, por ende, la pérdida lingüística.

Por último queríamos profundizar en el apartado de "Actitudes", esto es, en las razones afectivas o las intenciones para el futuro que se suelen traducir en la preferencia por el contacto con una $u$ otra lengua; por ejemplo, el deseo de reinmigración podría provocar un deseo de rodearse de hablantes de rumano o mientras que

\footnotetext{
${ }^{3}$ La idea la hemos tomado de algunas encuestas de usos lingüísticos referidas a las lenguas cooficiales de España, por ejemplo la realizada por el Centro Gallego de Investigaciones Sociológicas.
} 
la intención de quedarse definitivamente en España, se traduciría en la actitud contraria, preferir pasar más tiempo en contacto con hablantes de español.

Después intentamos ver las conexiones que, para los miembros de la comunidad rumana, existen entre la conservación de lengua rumana y la integración en la sociedad de acogida. Esto es, indagar en los prejuicios que suele haber sobre el bilingüismo entre los propios inmigrantes y ver si estos siguen vigentes. Tratamos de ver si hay contradicciones o grandes diferencias entre la opinión sobre el hecho general del futuro de la lengua y la opinión personal, esto es, si hay hablantes que se muestran pesimistas u optimistas por el futuro del rumano dentro de la comunidad pero que personalmente no piensan de la misma manera.

\section{Resultados del cuestionario}

\section{A) Uso}

- Lengua materna - lengua de instalación

El 100\% de los encuestados declaran tener el rumano como lengua materna. Ahora bien, el 90,9\% afirma que su única lengua materna es el rumano, mientras que el 1,81\% afirma tener además el húngaro como lengua materna y el 7,27\% declaran tener "otra" lengua materna además del rumano, sin especificar cuál.

Estos datos contrastan con la pregunta 12, ‘En qué lengua piensa?', que utilizamos para ver cuál es la lengua de instalación de los encuestados en el momento de responder al cuestionario. El 43,63\% responde que piensan en ambas lenguas, español y rumano. El porcentaje de los informantes que afirma pensar en español alcanza el 7,27\%, mientras los que responden que piensan en rumano son un $49^{\prime} 09 \%$.

\begin{tabular}{|l|l|l|l|}
\hline \multicolumn{2}{|c|}{ Lengua materna } & \multicolumn{2}{c|}{ Lengua principal } \\
\hline Rumano & $90^{\prime} 9 \%$ & Rumano & $49^{\prime} 09^{\prime} \%$ \\
\hline Rumano + Húngaro & $1^{\prime} 81 \%$ & Rumano / Español & $433^{\prime} 63 \%$ \\
\hline Rumano + Otra & $77^{\prime} 27 \%$ & Español & $7^{\prime} 27 \%$ \\
\hline
\end{tabular}

Tabla 7: Lengua materna y lengua principal de los informantes Resultados del 'Cuestionario sobre actitudes lingüísticas...'

Esto quiere decir que pese a declarar poseer el rumano como lengua materna, muchos individuos bilingües sienten que su lengua de instalación es actualmente o bien el español o bien el rumano y el español alternativamente. Estos datos apuntan pues hacia una penetración importante del español como lengua habitual de los miembros de la comunidad rumana. Curiosamente ninguna de las personas que declaraba tener otra lengua materna además del rumano la menciona como lengua en la que piensa, consecuencia quizás de un bilingüismo imperfecto en el país de origen. 
Para estudiar la lengua de instalación, hemos incluido además una pregunta indirecta dentro de la sección dedicada a los usos de la lengua, se trata del apartado "redactar notas personales". Para autores como Miquel Siguan, la lengua en la que nos dirigimos a nosotros mismos es nuestra lengua de instalación. Veamos los resultados del análisis de las respuestas para esta pregunta:

\begin{tabular}{|l|c|l|}
\hline & Promedio $^{4}$ & \multicolumn{1}{c|}{ Resultado } \\
\hline $\begin{array}{l}\text { Redactar notas persona- } \\
\text { les }\end{array}$ & 3'56 & $\begin{array}{l}\text { Tanto rumano como español / } \\
\text { Más rumano que español }\end{array}$ \\
\hline
\end{tabular}

Tabla 8: Lengua en que se redactan notas personales Resultados del 'Cuestionario sobre actitudes lingüísticas...'

Así pues, para el epígrafe 'redactar notas personales' el resultado se sitúa entre la alternancia de las lenguas y un uso inclinado más hacia el rumano que hacia el español como lengua de instalación.

- Ámbitos de uso

Cuando los hablantes emplean dos variedades lingüísticas, es obvio que no usan las dos en todas las circunstancias. En determinadas ocasiones utilizarán una de ellas y en otros momentos, la otra. La situación en la que se produce el acto comunicativo tiene una influencia importante a la hora de elegir la lengua: en casa, en el trabajo, en la calle con un desconocido, en el tiempo libre, etcétera. Estas situaciones en que se desarrollan unas determinadas funciones comunicativas se denominan ámbitos. Conviene asumir que lenguas diferentes pueden cumplir funciones diferentes en la vida de los hablantes bilingües y eso determina su elección lingüística, más allá de que su lengua tenga mayor o menor prestigio.

-Familia

\begin{tabular}{|l|l|l|}
\hline Con los padres & $4^{\prime} 9^{5}$ & Rumano \\
\hline Con la pareja & $3^{\prime} 51$ & $\begin{array}{l}\text { Tanto rumano como español / } \\
\text { Más rumano que español }\end{array}$ \\
\hline Con otros familiares & $4^{\prime} 49$ & $\begin{array}{l}\text { Rumano / } \\
\text { Más rumano que español }\end{array}$ \\
\hline
\end{tabular}

Tabla 9: Lengua utilizada en el ámbito familiar. Resultados del 'Cuestionario sobre actitudes...'

\footnotetext{
${ }^{4}$ desviación de la muestra: 0’516

${ }^{5}$ Desviación típica: 0’36
} 
La diferencia de un punto entre la categoría 'con la pareja' y las otras dos categorías se debe principalmente a que en muchas ocasiones los padres y la familia se encuentran en Rumanía y por ello es obvio que la lengua de comunicación empleada será el rumano. La lengua preferida para hablar con la pareja cambia ligeramente y aunque se inclina hacia el lado rumano, el español gana fuerza porque podemos encontrar parejas mixtas entre rumanos y españoles o rumanos y otras nacionalidades, que encuentran en el español el nexo de unión.

-Trabajo
\begin{tabular}{|l|l|l|}
\hline Con el jefe & $1^{\prime} 62^{6}$ & $\begin{array}{l}\text { Español / } \\
\text { Más español que rumano }\end{array}$ \\
\hline Con los compañeros & $2^{\prime} 12$ & Más español que rumano \\
\hline
\end{tabular}

Tabla 10: Lengua utilizada en el ámbito laboral Resultados del 'Cuestionario sobre actitudes...'

En ambos casos observamos que la lengua más utilizada es el español, de hecho solo en una ocasión un informante declara hablar únicamente rumano con su jefe. Por ello podemos concluir que nuestros encuestados trabajan para españoles y principalmente con españoles, a pesar de que la lengua rumana está también presente en el lugar de trabajo para comunicarse con compañeros de la misma nacionalidad.

\section{-Amigos}

\begin{tabular}{|l|l|l|}
\hline promedio general & $3^{\prime} 38^{7}$ & Tanto rumano como español \\
\hline
\end{tabular}

Tabla 11: Lengua utilizada con los amigos.

Resultados del 'Cuestionario sobre actitudes...'

Los resultados de esta sección confirman que en general los miembros de la comunidad rumana que han participado en esta investigación desarrollan su círculo de amistades en un contexto bilingüe, en el cual están presentes tanto la lengua española como la lengua rumana. Para intentar profundizar más decidimos valorar los datos por géneros para ver si se mantenía el promedio. No obstante, los resultados fueron muy parecidos al promedio general, de lo cual podemos deducir que el comportamiento de hombres y mujeres a la hora de realizar la elección lingüística es semejante en este ámbito comunicativo, si bien en el caso de los hombres hay una leve tendencia a preferir el rumano.

\footnotetext{
${ }^{6}$ Desviación típica: 1'12

${ }^{7}$ Desviación típica: 1'22
} 


\begin{tabular}{|l|l|l|}
\hline Mujeres & $3^{\prime} 37^{8}$ & Tanto rumano como español \\
\hline Hombres & $3^{\prime} 69^{9}$ & $\begin{array}{l}\text { Tanto rumano como español / } \\
\text { Más rumano que español }\end{array}$ \\
\hline
\end{tabular}

Tabla 12: Lengua utilizada con los amigos, por sexos.

Resultados del 'Cuestionario sobre actitudes...'

-Instituciones

\begin{tabular}{|l|l|l|}
\hline Promedio general & $1 ' 35$ & Español \\
\hline
\end{tabular}

Tabla 12: Lengua utilizada en el ámbito institucional

Resultados del 'Cuestionario sobre actitudes...'

En este ámbito esperaríamos una puntuación muy cercana al 1, es decir, al uso exclusivo del español, sin embargo la puntuación obtenida es algo superior a esta cifra. Las razones para explicar este resultado podrían ser por ejemplo la existencia de traductores en algunas instituciones, como por ejemplo el sistema educativo o bien el hecho de encontrar compatriotas trabajando para algunos ayuntamientos, especialmente en el área de inmigración ${ }^{10}$. Ahora bien, el $87 \%$ de las respuestas apuntaron hacia la opción "español".

-Tiempo libre

\begin{tabular}{|c|c|c|}
\hline Tipo de actividad & $\begin{array}{l}\text { Promedio } \\
\text { general }\end{array}$ & Lengua preferida \\
\hline lectura : libros & $2^{\prime} 73$ & $\begin{array}{l}\text { Más español que rumano / } \\
\text { Tanto rumano como español }\end{array}$ \\
\hline lectura : revistas & $2 ’ 28$ & Más español que rumano \\
\hline lectura : periódicos & $2 ’ 33$ & Más español que rumano \\
\hline música & $2^{\prime} 62$ & $\begin{array}{l}\text { Más español que rumano / } \\
\text { Tanto rumano como español }\end{array}$ \\
\hline cine & $1 ' 87$ & $\begin{array}{l}\text { Más español que rumano / } \\
\text { Español }\end{array}$ \\
\hline radio & $1 ' 83$ & $\begin{array}{l}\text { Más español que rumano / } \\
\text { Español }\end{array}$ \\
\hline televisión & $2 ’ 2$ & Más español que rumano \\
\hline
\end{tabular}

\footnotetext{
${ }^{8}$ Desviación típica: 1 '15

${ }^{9}$ Desviación típica: 1 ' 44

${ }^{10}$ Información ofrecida por los propios informantes.
} 


\begin{tabular}{|l|l|l|}
\hline Internet & $2 ’ 25$ & Más español que rumano \\
\hline TOTAL & $2 ’ 26$ & Más español que rumano \\
\hline
\end{tabular}

Tabla 13: Lengua utilizada en el ámbito del ocio

Resultados del 'Cuestionario sobre actitudes...'

El análisis de los resultados que se refieren a las actividades de ocio que conllevan el uso de una u otra lengua sitúa al español por delante del rumano como lengua de uso por parte del colectivo rumano. La principal razón de esta sustitución suele ser la motivación instrumental o integrativa para aprender el idioma y en numerosas ocasiones las actividades de ocio sirven para aprender o mejorar el español. Nosotros apuntamos igualmente hacia la mayor disponibilidad de medios de comunicación en español, sobre todo libros, revistas, periódicos y cadenas de televisión. Sin embargo sabemos que muchos hogares constituidos por familias rumanas disponen de televisión vía satélite y de diferentes abonos a las cadenas de televisión rumanas, aunque no por ello la puntuación del apartado 'televisión' es superior a las otras. Sorprende también que el acceso a Internet se lleve a cabo preferentemente en español.

La puntuación de la actividad 'lectura: libros' es la más alta, a pesar de que no llega al número 3, la puntuación que indica una utilización de ambas lenguas; esto es un signo indicativo de que el contacto con la literatura en rumano puede estar en peligro y, por lo tanto, la lengua rumana en Madrid podría perder el referente de la norma culta literaria, con las consecuencias lingüísticas que esto podría acarrear.

Los resultados para el grupo de edad comprendido entre los 18 y los 25 años no difieren esencialmente de los datos generales, el promedio total de las actividades de ocio se inclina un poco más hacia el español. Sin embargo, la puntuación de lectura de libros y música es ligeramente superior al promedio general; notemos que también entre los más jóvenes son estas dos actividades las que presentan una puntuación más alta, es decir, se emplea el rumano con más frecuencia.

\begin{tabular}{|l|c|l|}
\hline \multicolumn{1}{|c|}{ Tipo de actividad } & $\begin{array}{c}\text { Promedio del } \\
\text { grupo de edad } \\
18-25\end{array}$ & \multicolumn{1}{|c|}{ Lengua preferida } \\
\hline lectura : libros & $2^{\prime} 86$ & $\begin{array}{l}\text { Más español que rumano / } \\
\text { Tanto rumano como español }\end{array}$ \\
\hline lectura : revistas & $2 ’ 25$ & Más español que rumano \\
\hline lectura : periódicos & $2 ’ 25$ & Más español que rumano \\
\hline música & $2^{\prime} 75$ & $\begin{array}{l}\text { Más español que rumano / } \\
\text { Tanto rumano como español }\end{array}$ \\
\hline cine & $1{ }^{\prime} 5$ & Español \\
\hline radio & $1^{\prime} 75$ & Más español que rumano \\
\hline televisión & $1^{\prime} 83$ & Más español que rumano \\
\hline
\end{tabular}




\begin{tabular}{|c|c|l|}
\hline Internet & 1'54 & Español \\
\hline TOTAL & $2 ’ 09$ & Más español que rumano \\
\hline
\end{tabular}

Tabla 14: Lengua utilizada en el ámbito del ocio por el grupo de edad 18-25 años. Resultados del 'Cuestionario sobre actitudes...'

\section{- Uso de la lengua e integración social}

El último aspecto que queríamos tratar en esta sección consiste en la percepción extendida entre los componentes de la comunidad de habla inmigrante de que existe una asociación entre utilizar más la lengua de la sociedad de acogida y una mejor integración en la misma. La mayoría de los informantes respondieron de manera afirmativa a la pregunta planteada, es decir, hablar más español que rumano significa para ellos integrarse mejor en la sociedad de acogida. Lo que sí resulta sorprendente es el desglose de los resultados por sexos: entre los hombres más de las tres cuartas partes de los encuestados apoyan el sí, mientras que esta cifra apenas supera el $50 \%$ entre las mujeres.

\begin{tabular}{|l|c|c|}
\hline & si & $n o$ \\
\hline Promedio general & $59^{\prime} 26 \%$ & $40^{\prime} 73 \%$ \\
\hline Mujeres & $51^{\prime} 35 \%$ & $48^{\prime} 64 \%$ \\
\hline Hombres & $76^{\prime} 47 \%$ & $23^{\prime} 52 \%$ \\
\hline
\end{tabular}

Tabla 15: Relación entre hablar español y mejor integración, en general y por sexos.

Resultado del 'Cuestionario sobe actitudes...'

Así pues los hombres encuestados ven una relación clara entre relegar el rumano a un segundo plano e integrarse en la sociedad; entre las mujeres esta relación no es evidente, fruto quizás, como hemos mencionado en otras ocasiones a lo largo de estas páginas, de un mayor conservadurismo lingüístico.

Hemos también querido saber si los informantes creen que conservar la lengua en la segunda generación podría provocar problemas de integración, a través de la pregunta '¿Cree que si sus hijos hablan rumano tendrán más problemas para integrarse en la sociedad madrileña?'. Esto es, le hemos dado la vuelta a la pregunta anterior, mencionando en esta ocasión la palabra "rumano" en el enunciado de la pregunta.

\begin{tabular}{|l|c|c|}
\hline & \multicolumn{1}{|c|}{ si $^{\prime}$} & $n o$ \\
\hline Promedio general & $27^{\prime} 26 \%$ & $72^{\prime} 73 \%$ \\
\hline Mujeres & $31^{\prime} 57 \%$ & $68^{\prime} 42 \%$ \\
\hline Hombres & $17^{\prime} 65 \%$ & $82{ }^{\prime} 35 \%$ \\
\hline
\end{tabular}

Tabla 16: Relación entre hablar rumano y problemas en la integración Resultado del 'Cuestionario sobe actitudes...' 
Ahora la respuesta es mucho más evidente, más del 70\% de los informantes no creen que hablar rumano sea un problema para la integración, sin embargo el $60 \%$ indicaba anteriormente que era mejor hablar más español a la hora de integrarse. ¿Estamos ante una contradicción? No necesariamente, se trata más bien de la aceptación de una situación diglósica: el español debe ser la lengua principal para conseguir la integración, aunque el rumano no "molesta" tampoco. O quizás sí "molesta", ya que una cuarta parte de los encuestados admite que hablar rumano puede ser un problema para la integración. El componente afectivo también juega un papel relevante en esta ocasión, la mayoría de los informantes no quiere asociar su idioma con problemas de ningún tipo.

\section{B) ACTITUDES}

\section{- $\quad$ Las actitudes de los hablantes rumanos}

Las situaciones de inmigración suelen traducirse desgraciadamente en actitudes y comportamientos negativos de los miembros de la comunidad de habla inmigrante hacia su propia lengua. Los inmigrantes tienden a valorar de manera negativa su idioma, a considerarlo inferior a la lengua mayoritaria de la sociedad, ven su propia lengua poco o nada útil e incluso como una barrera para la integración, por ello los contextos de lenguas en contacto por inmigración progresan en numerosas ocasiones hacia la sustitución y el abandono de la lengua minoritaria.

Esta actitud merecería más bien el nombre de "autoprejuicio" y en más de una ocasión ha sido designada con expresiones tales como "autoodio" o "deslealtad lingüística. En resumidas cuentas, esta valoración negativa no es otra cosa que una forma de justificar el adiós a la lengua, el sentimiento de que ya no sirve. (Tusón 1997: 26)

En cualquier caso, es importante tener en cuenta la distancia psicológica y social entre las dos lenguas que protagonizan la situación de contacto. Así el inmigrante que pretende aprender la lengua de la sociedad de acogida tendrá más éxito si no presenta actitudes negativas ante ésta o bien si no percibe actitudes negativas hacia sí mismo por parte del grupo dominante.

\section{- Transmisión a los hijos (Actitudes)}

En este apartado analizaremos los resultados referidos a la transmisión de la lengua rumana a la segunda generación, un hecho de vital importancia para el mantenimiento y supervivencia de una lengua minoritaria en el contexto de la inmigración. De acuerdo con la información aportada por los encuestados, el rumano es la lengua preferida para hablar con los hijos, por lo tanto, la transmisión a la segunda generación no se interrumpe en el ámbito familiar. Ahora bien, entre 
los informantes que no tienen hijos, gana fuerza la idea del bilingüismo en casa, es decir, hablar con los hijo tanto en una como en otra lengua.

\begin{tabular}{|l|c|l|}
\hline Tipo de informante & Promedio & Lengua preferida \\
\hline Informantes con hijos & $4 ’ 06$ & Más rumano que español \\
\hline Informantes sin hijos & $3 ’ 53$ & $\begin{array}{l}\text { Tanto rumano como español / } \\
\text { Más rumano que español }\end{array}$ \\
\hline
\end{tabular}

Tabla 17: Lengua preferida para transmitir a los hijos.

Resultados del 'Cuestionario sobre actitudes...'

La idea de emplear las dos lenguas en la comunicación con los hijos puede responder más bien a un deseo de equilibrio entre los dos idiomas más que a una situación real, de hecho, si un progenitor rumano decide emplear tanto su lengua primera como la lengua de la sociedad de acogida, cabe la posibilidad de que la lengua primera, el rumano, vaya perdiendo frecuencia de uso en el propio contexto familiar, lo cual podría ocasionar una sustitución lingüística. Además existe el riesgo de que se transmita un español incorrecto, con errores que podrían quedar fosilizados en la interlengua de la segunda generación.

$\mathrm{El}$ análisis por sexos depara los siguientes resultados:

\begin{tabular}{|l|c|l|}
\hline Con hijos & Puntuación & \multicolumn{1}{c|}{ Lengua preferida } \\
\hline Mujeres & 4 '25 & Más rumano que español \\
\hline Hombres & $3^{\prime} 6$ & $\begin{array}{l}\text { Tanto rumano como español / } \\
\text { Más rumano que español }\end{array}$ \\
\hline
\end{tabular}

Tabla 18: Transmisión de la lengua a la segunda generación, por sexos. (Informantes con hijos) Resultados del 'Cuestionario sobre actitudes...'

\begin{tabular}{|l|c|l|}
\hline Sin hijos & Puntuación & \multicolumn{1}{c|}{ Lengua preferida } \\
\hline Mujeres & 3'59 & $\begin{array}{l}\text { Tanto rumano como español / } \\
\text { Más rumano que español }\end{array}$ \\
\hline Hombres & 3'41 & Tanto rumano como español \\
\hline
\end{tabular}

Tabla 19: Transmisión de la lengua a la segunda generación, por sexos. (Informantes sin hijos) Resultados del 'Cuestionario sobre actitudes...'

De estos cuadros podemos deducir que, pese a la relativa semejanza de los resultados para hombres y mujeres, las mujeres presentan una puntuación más alta, es decir, eligen emplear el rumano en mayor medida que los hombres, tanto entre el grupo que tiene ya hijos como en el grupo que no los tiene. Asimismo, las puntuaciones tanto para hombres como para mujeres con hijos son mayores que las de 
los hombres y mujeres que no los tienen, quienes sin duda son más partidarios de emplear las dos lenguas en casa con la segunda generación.

En los apartados de nuestro cuestionario que se referían al uso del rumano con los hijos muchos de los informantes incluyeron comentarios personales. Entre ellos, hay razones de todo tipo para defender el uso del rumano, que vamos a intentar clasificar:

-Razones de identidad, son las más numerosas:

"para no perder nuestras raíces"

"porque es mi idioma materno"

"a lo mejor el niño cuando llegue a la madurez desea regresar a Rumanía" (intención de reinmigración en el país de origen, se está documentando una notable desconexión entre los hijos de inmigrantes rumanos criados en España cuando retornan a Rumanía) "para que no se pierda el idioma"

"para conservar nuestra identidad"

"porque soy rumano"

"aun soy rumana"

"porque son rumanos para siempre"

"son rumanos, tienen que hablar rumano"

-Beneficios del bilingüismo:

"para que sepan varios idiomas"

"para que sean bilingües"

"siendo un idioma muy complicado les vendría bien acostumbrarse a él" mujer, sin hijos (estereotipo lingüístico sobre la dificultad a priori de los idiomas)

-Ventajas culturales:

"formar parte de dos culturas es enriquecedor"

"considero que el idioma es un elemento cultural importante sobre todo en los hijos de parejas mixtas" (informante: mujer casada, con hijos)

-Competencia lingüística:

"porque te sale natural hablar con ellos en rumano"

"porque es el único idioma que conozco bien" (se evita así la tentación de caer en el semilingüismo si el progenitor decidiera transmitir a los hijos una lengua que no domina con fluidez)

También encontramos argumentos a favor de la utilización de los dos idiomas para la transmisión a la segunda generación. En teoría este modelo es "arriesgado" para la supervivencia de la lengua, salvo en el caso de parejas mixtas, ya que la implantación de la lengua dominante en el ámbito familiar es una de las principales amenazas para el mantenimiento de la lengua minoritaria: 
-Beneficios del bilingüismo (en este caso los informantes creen que las lenguas se aprenden solamente en el ámbito familiar y quizás por eso eligen hablar con los hijos en los dos idiomas, olvidando así el papel de la escuela y del contexto social de Madrid, donde la lengua imperante es el español, idioma que no necesitaría ser usado en el ámbito familiar para ser adquirido por los niños):

"aprender dos idiomas es una ventaja en la vida"

"es mejor hablar con ellos en dos idiomas"

-Razones culturales / personales:

"por costumbre"

"porque los dos idiomas forman parte de mi identidad" (es importante cuando un hablante miembro de la comunidad rumana se considera a sí mismo como bicultural y por ello decide transmitir sus dos idiomas a la siguiente generación, sin embargo el riesgo de sustitución lingüística es elevado)

"porque es más cómodo"

"porque los dos idiomas son importantes"

-Nexo de unión con la identidad rumana:

"porque ahora vivimos en España y el rumano porque quizás regresamos a Rumanía" "para integrarse en España pero que no pierda la relación con sus abuelos"

Podemos distinguir también un tercer tipo de actitudes en torno a la elección de una $\mathrm{u}$ otra lengua para transmitir a los hijos: se trata de aquellos miembros de la comunidad de habla rumana de Madrid que optan por transmitir la lengua dominante el español. Hay diferentes razones que apoyan esta decisión, la más habitual será la existencia de un matrimonio o pareja mixta, en cuyo contexto se haya decidido emplear el español como vehículo de comunicación.

-Pareja mixta:

"Porque mi pareja es español y en nuestro entorno todo el mundo habla español"

-Contexto geográfico:

"porque mi hija nació en España"

\section{- Actitudes hacia la transmisión de la lengua}

Queríamos comparar los datos anteriores sobre la elección de una determinada variedad lingüística mediante una simple pregunta, la referida a si les gustaría que sus hijos hablaran correctamente rumano.

\begin{tabular}{|c|c|c|}
\hline & $s i$ & $n o$ \\
\hline Porcentaje de informantes & $98^{\prime} 18 \%$ & $0 \%$ \\
\hline
\end{tabular}

Tabla 20: Actitudes ante el uso del rumano por parte de sus hijos

Resultado del 'Cuestionario sobe actitudes...' 
Hemos obtenido un resultado relativamente sorprendente: casi todos los informantes expresan su deseo de que los hijos dominen la lengua rumana sin embargo este porcentaje no es tan alto cuando se les pregunta, como hicimos anteriormente, por la lengua que emplean con los hijos. Es decir, les gustaría que sus hijos hablaran rumano pero muchos de ellos ni siquiera emplean esta lengua en casa. Se trataría así pues de una actitud del tipo "Todo para el rumano pero sin el rumano", comportamiento muy parecido al observado por Iglesias en su estudio sobre los usos sociales del gallego, los hablantes se muestran a favor de que la lengua sea hablada por la segunda generación pero ellos no se implican en la transmisión.

En cuanto a la presencia del rumano en la escuela pública madrileña, más de tres cuartas partes de los encuestados aseguran que es necesario que los hijos de rumanos aprendan la lengua primera en la escuela. El porcentaje de quienes se muestran en contra de esta medida apenas sobrepasa el $10 \%$.

\begin{tabular}{|l|l|}
\hline Sí, es necesario & $78^{\prime} 18 \%$ \\
\hline Sí, pero no es lo más importante & $10^{\prime} 91 \%$ \\
\hline No, se aprende en la familia & $5^{\prime} 45 \%$ \\
\hline No, deben aprender solo español & $5^{\prime} 45 \%$ \\
\hline
\end{tabular}

Tabla 22: Actitudes ante la presencia del rumano en la escuela Resultado del 'Cuestionario sobe actitudes...'

En este apartado consideraremos así mismo los aspectos afectivos de la elección lingüística a través de los resultados de la pregunta "¿Prefiere pasar el tiempo con gente que hable rumano o con gente que hable español?":

\begin{tabular}{|l|l|}
\hline Con gente que hable rumano & $16^{\prime} 66 \%$ \\
\hline Con gente que hable español & $11^{\prime} 11 \%$ \\
\hline Me da igual & $72^{\prime} 22 \%$ \\
\hline
\end{tabular}

Tabla 23: Actitudes ante la elección de interlocutores de una u otra lengua. Resultado del 'Cuestionario sobe actitudes...'

Es complicado extraer algún dato relevante en el análisis por edades de estos dos grupos, ya que la muestra que manejamos es demasiado pequeña y por ello, poco representativa. En todo caso la preferencia mayoritaria de todos los grupos de edad es la tercera opción, estar con hablantes de cualquiera de las dos lenguas.

Cuando se les pide que puntúen de 1 a 5 (la puntuación 1 representa 'en total desacuerdo' y la puntuación 5, 'totalmente de acuerdo'), la actitud de los padres rumanos que no hablan rumano con sus hijos, obtenemos los siguientes resultados: 


\begin{tabular}{|l|l|}
\hline Promedio general & $1^{\prime} 92$ \\
\hline Mujeres & $1^{\prime} 83$ \\
\hline Hombres & $2^{\prime} 125$ \\
\hline
\end{tabular}

Tabla 24: Actitud ante los padres que no emplean el rumano con sus hijos

Resultado del 'Cuestionario sobe actitudes...'

En general los encuestados reprueban la actitud de quienes no transmiten la lengua rumana a sus hijos, las mujeres en mayor medida que los hombres, que se consideran tradicionalmente más propensos a los fenómenos de sustitución de lenguas y menos conservadores. Este dato de defensa del rumano y "condena" hacia quienes no emplean la lengua primera con los hijos contrasta con los resultados anteriores que apuntaban hacia el uso de las dos lenguas con los hijos.

\section{- Actitudes de futuro: mantenimiento o sustitución lingüística}

Concretamos mediante la pregunta '¿cree que dentro de veinte años los rumanos de Madrid seguirán hablando principalmente rumano?' La respuesta más frecuente es la que corresponde a la situación de mantenimiento de la lengua menos optimista, es decir, que pocas familias conserven el rumano. De todas formas, las tres respuestas que corresponden a la lealtad lingüística en mayor o menor grado van por delante de la única respuesta que da cuenta de una situación de sustitución lingüística.

\begin{tabular}{|l|l|}
\hline Sí, igual que hoy & $29^{\prime} 62 \%$ \\
\hline Sí, pero solo las personas mayores & $22^{\prime} 22 \%$ \\
\hline Sí, pero muy pocas familias & $33^{\prime} 33 \%$ \\
\hline No, hablarán más español & $14^{\prime} 81 \%$ \\
\hline
\end{tabular}

Tabla 25: Valoración de los informantes sobre el mantenimiento de la lengua en el futuro Resultado del 'Cuestionario sobe actitudes...'

Si profundizamos en el aspecto afectivo del futuro del rumano tampoco encontramos grandes sorpresas, la inmensa mayoría de los informantes opina que es importante mantener la lengua rumana como símbolo de la identidad colectiva del grupo, tal y como ya habíamos apuntado anteriormente en el caso de la transmisión de la lengua a la siguiente generación. La suma de quienes son indiferentes ante el futuro del idioma y de quienes defienden la sustitución lingüística no llega al $10 \%$ del total: 


\begin{tabular}{|l|l|}
\hline Sí, me parece importante conservar nuestra identidad & $87^{\prime} 04 \%$ \\
\hline Sí, pero no me parece muy importante & $3{ }^{\prime} 7 \%$ \\
\hline No, hay que sustituir el rumano por el español & $3^{\prime} 7 \%$ \\
\hline Me da igual & $5^{\prime} 55 \%$ \\
\hline
\end{tabular}

Tabla 26: Opinión de los informantes sobre el mantenimiento o la sustitución del rumano Resultado del 'Cuestionario sobe actitudes...'

Si comparamos el aspecto afectivo con la pregunta más objetiva sobre el futuro de la lengua observamos una enorme diferencia de cifras. Mientras que a casi todos los informantes les parece necesario conservar el idioma, solo a un $30 \%$ le parece que el futuro del rumano será parecido al presente. Es decir, les gustaría que la lengua se mantuviera pero reconocen que la realidad podría ser mucho menos optimista.

En definitiva, a lo largo de estas páginas hemos tratado de caracterizar, mediante el análisis de los datos extraídos de la investigación empírica sobre el terreno, un fenómeno sociolingüístico reciente, el de las lenguas rumana y española en una situación de contacto en el área metropolitana de Madrid, hecho sobre el cual existe todavía muy poca bibliografía, pero que pone en evidencia la importancia de la inmigración y de lo urbano como escenarios del contacto lingüístico.

\section{Bibliográfía}

Instituto Nacional de Estadística (2009), Padrón municipal. Datos de población, referidos al 1 de enero de 2009.

SiguAN, Miquel (2001), Bilingüismo y lenguas en contacto en España. Madrid: Alianza Editorial.

Tusón, Jesús (1997), Los prejuicios lingüisticos, Barcelona: Octaedro. 